\title{
Solving Pseudomonotone Variational Inequalities and Pseudoconvex Optimization Problems Using the Projection Neural Network
}

\author{
Xiaolin Hu, Student Member, IEEE, and Jun Wang, Senior Member, IEEE
}

\begin{abstract}
In recent years, a recurrent neural network called projection neural network was proposed for solving monotone variational inequalities and related convex optimization problems. In this paper, we show that the projection neural network can also be used to solve pseudomonotone variational inequalities and related pseudoconvex optimization problems. Under various pseudomonotonicity conditions and other conditions, the projection neural network is proved to be stable in the sense of Lyapunov and globally convergent, globally asymptotically stable, and globally exponentially stable. Since monotonicity is a special case of pseudomononicity, the projection neural network can be applied to solve a broader class of constrained optimization problems related to variational inequalities. Moreover, a new concept, called componentwise pseudomononicity, different from pseudomononicity in general, is introduced. Under this new concept, two stability results of the projection neural network for solving variational inequalities are also obtained. Finally, numerical examples show the effectiveness and performance of the projection neural network.
\end{abstract}

Index Terms-Componentwise pseudomonotone variational inequality, global asymptotic stability, projection neural network, pseudoconvex optimization, pseudomonotone variational inequality.

\section{INTRODUCTION}

$\mathbf{V}$ ARIATIONAL INEQUALITY (VI) can be viewed as a natural framework for unifying the treatment of equilibrium problems, and hence has many applications such as the analysis of piecewise-linear resistive circuits, bimatrix equilibrium, economic equilibrium modeling, traffic network equilibrium modeling, elasticity, and structural analysis [1]-[6]. An explosive growth in theoretical advances, algorithmic development, and applications across many disciplines have been witnessed over decades. Various numerical procedures have been presented for solving VI with the assumption that the underlying operator is monontone [1], [4], [7]-[10]. When the underlying operator is pseudomonotone, a weaker condition than monotonicity, several iterative algorithms for solving it are also available [11]-[13].

In many engineering applications such as signal processing, system identification, and robot motion control [14], [15] realtime solutions are often desired. But these problems may be high

Manuscript received August 24, 2005; revised March 31, 2006. This work was supported by the Hong Kong Research Grants Council under Grant CUHK4165/ 03E.

The authors are with the Department of Automation and Computer-Aided Engineering, The Chinese University of Hong Kong, Shatin, New Territories, Hong Kong, China (e-mail: jwang@acae.cuhk.edu.hk).

Digital Object Identifier 10.1109/TNN.2006.879774 in dimension and dense in structure. Conventional numerical methods, however, may not be efficient in such occasions due to stringent requirement on computing time. A promising approach to handle these problems is to employ artificial neural networks based on circuit implementation [16], [17]. Unlike other parallel algorithms, neural networks can be implemented physically in designated hardware such as application-specific integrated circuits where the optimization procedure is truly parallel and distributed. As a result, the neural network approach for optimization, control, and signal processing received tremendous interests.

In the past two decades, the theory, methodology, and applications of recurrent neural networks for optimization have been widely investigated since the seminal work of Hopfield and Tank [16], [17] (see [18]-[23] and references therein). Numerous neural network models have been developed, from the earlier proposals such as the penalty method-based neural network proposed by Kennedy and Chua [18], the switched-capacitor neural network by Rodríguez-Vázquez et al. [19] and the deterministic annealing neural network by Wang [20], to the latest development such as Xia and Wang et al. [21]-[23]. Regarding solving VI, a recurrent neural network, called projection neural network is developed in [24]-[28]. The global convergence of the neural networks are always ensured by various monotonicity conditions on the linear or nonlinear operator together with other conditions except in [26] (In [26], the convergence results are expressed in terms of the Lyapunov diagonally stability concept). Recently, Noor [31] claimed that the projection neural network can be used to solve VI by only requiring the pseudomonotonicity of the operator. Although his work is incomplete (a counter example to his statement will be shown later), it suggests a possibility to solve pseudomonotone VI by using neural networks. In this paper, we show that the projection neural network presented in [24]-[28] can indeed solve the pseudomonotone VI under suitable assumptions. As a consequence, the scope of the related optimization problems can be enlarged from convex optimization problems to pseudoconvex optimization problems.

The remainder of the paper is organized as follows. The problem formulations and related preliminaries are presented in Section II. In Section III, we discuss the stability of the projection neural network for solving the pseudomonotone VI with box-type or sphere-type constraints and related pseudoconvex optimization problems. The global convergence, global asymptotic stability, and global exponential stability of the neural network are studied under different conditions. In Section IV, 
a set of new concepts, componentwise pseudomonotonicities (CPM), are introduced first, which follows the discussion on the stability of the projection neural network for solving CPM VI. Illustrative examples are presented in Section V. Section VI gives the conclusion of the paper.

\section{PROBlem Formulation AND PRELIMINARIES}

Let $R^{n}$ be the Euclidean space, $\Omega$ be a closed convex set in $R^{n}$, and $F(x)$ be a continuous vector-valued function from $R^{n}$ to $R^{n}$. Consider the problem of finding $x^{*} \in \Omega$ such that

$$
F\left(x^{*}\right)^{T}\left(x-x^{*}\right) \geq 0 \quad \forall x \in \Omega .
$$

Problem (1) is called variational inequality, denoted by $\operatorname{VI}(F, \Omega)$. It is well known that $x^{*} \in \Omega$ is a solution of $\mathrm{VI}(F, \Omega)$ if and only if it satisfies the relation

$$
x^{*}=P_{\Omega}\left(x^{*}-\alpha F\left(x^{*}\right)\right)
$$

where $\alpha>0$ is a constant and $P_{\Omega}: R^{n} \rightarrow \Omega$ is a projection operator defined by

$$
P_{\Omega}(x)=\arg \min _{y \in \Omega}\|x-y\|
$$

In (3), $\|\cdot\|$ denotes the $l_{2}$-norm of $R^{n}$.

In view of the equivalent formulation of $\operatorname{VI}(F, \Omega)$ in $(2)$, the following recurrent neural network, called projection neural network for solving (1) is developed in [24]-[28]

$$
\frac{d x}{d t}=\lambda\left\{-x+P_{\Omega}(x-\alpha F(x))\right\}
$$

where $\lambda>0$ and $\alpha>0$ are two scaling factors. Note $x^{*}$ is a solution of $\operatorname{VI}(F, \Omega)$ if and only if it is an equilibrium point of the above neural network. It is shown that under some mile conditions, the projection neural network can be used to solve monotone VI. In the current paper, we will show that this neural network is in addition capable of solving pseudomonotone VI. One of the merits of this neural network is its simplicity compared to the neural network studied in [29] and [30] for solving pseudomonotone VI.

It is seen that the projection neural network (4) involves a projection operator $P_{\Omega}(\cdot)$. In general, computing the projection of a point onto a convex set $\Omega$ is itself a complex optimization problem. However, if $\Omega$ is a box set or a sphere set, the calculation is straightforward. For example, if $\Omega=\left\{x \in R^{n} \mid l_{i} \leq\right.$ $\left.x_{i} \leq u_{i}, \forall i=1, \ldots, n\right\}$, then

$$
P_{\Omega}\left(x_{i}\right)= \begin{cases}l_{i}, & x_{i}<l_{i} \\ x_{i}, & l_{i} \leq x_{i} \leq u_{i} \\ u_{i}, & x_{i}>u_{i}\end{cases}
$$

Note $u_{i}$ might be $+\infty$ and $l_{i}$ might be $-\infty$. If $\Omega=\{x \in$ $\left.R^{n} \mid\|x-c\| \leq r, r>0\right\}$ where $c \in R^{n}$ and $r \in R$ are two constants. Then

$$
P_{\Omega}(x)=\left\{\begin{array}{ll}
x, & \|x-c\| \leq r \\
c+\frac{x-c}{\|x-c\|}, & \|x-c\|>r
\end{array} .\right.
$$

For the convenience of later discussion, it is necessary to introduce several notations, definitions, lemmas, and assumptions. In what follows, $\|\cdot\|$ denotes the $l_{2}$-norm of $R^{n}$; and $\Omega^{*}$ denotes the solution set of (1) or (2). If a function $g: R^{n} \rightarrow R$, then $\nabla g \in R^{n}$ stands for its gradient; if a function $G: R^{n} \rightarrow R^{m}$, $\nabla G \in R^{m \times n}$ stands for its Jacobian matrix.

Definition 1: A function $F: R^{n} \rightarrow R^{n}$ is said to be Lipschitz continuous with constant $L$ on the set $\Omega$ if, for every pair of points $x, y \in \Omega$

$$
\|F(x)-F(y)\| \leq L\|x-y\| \text {. }
$$

$F$ is said to be locally Lipschitz continuous on $\Omega$ if each point of $\Omega$ has a neighborhood $D_{0} \subset \Omega$ such that (5) holds for every pair of points $x, y \in D_{0}$.

Definition 2: A function $F: R^{n} \rightarrow R^{n}$ is said to be pseudomonotone on $\Omega$ if, for every pair of distinct points $x, y \in \Omega$

$$
F(x)^{T}(y-x) \geq 0 \Longrightarrow F(y)^{T}(y-x) \geq 0 .
$$

$F$ is said to be strictly pseudomonotone on $\Omega$ if, for every pair of distinct points $x, y \in \Omega$

$$
F(x)^{T}(y-x) \geq 0 \Longrightarrow F(y)^{T}(y-x)>0
$$

and strongly pseudomonotone on $\Omega$ if there exists a constant $\gamma>0$ such that for every pair of points $x, y \in \Omega$

$$
F(x)^{T}(y-x) \geq 0 \Longrightarrow F(y)^{T}(y-x) \geq \gamma\|x-y\|^{2} .
$$

The aforementioned definitions of pseudomonotonicity are easily seen as listed in an order from weak to strong. Moreover, the pseudomonotonicity is a generalization of monotonicity, the definition of which can be found in [27]. Clearly, monotonicity implies pseudomononicity, strict monotonicity implies strict pseudomononicity, and strong monotonicity implies strong pseudomononicity, but not vice versa.

Definition 3: A differentiable function $f: R^{n} \rightarrow R$ is pseudoconvex on $\Omega$ if for every pair of distinct points $x, y \in \Omega$

$$
\nabla f(x)^{T}(y-x) \geq 0 \Longrightarrow f(y) \geq f(x) .
$$

The function $f$ is said to be strictly pseudoconvex on $\Omega$ if for every pair of distinct points $x, y \in \Omega$

$$
\nabla f(x)^{T}(y-x) \geq 0 \Longrightarrow f(y)>f(x)
$$

and strongly pseudoconvex on $\Omega$ if there exists a constant $\beta>0$ such that for every pair of points $x, y \in \Omega$

$$
\nabla f(x)^{T}(y-x) \geq 0 \Longrightarrow f(y) \geq f(x)+\beta\|y-x\|^{2} .
$$

It is shown in [32] that a differentiable function is pseudoconvex and strictly pseudoconvex if and only if its gradient is a pseudomonotone and strictly pseudomonotone mapping, respectively. Moreover, if its gradient is strongly pseudomonotone, the function is strongly pseudoconvex; however, the converse is not true [33]. The following well-known result reveals the relationship between pseudoconvex optimization and VI [1], [4]. 


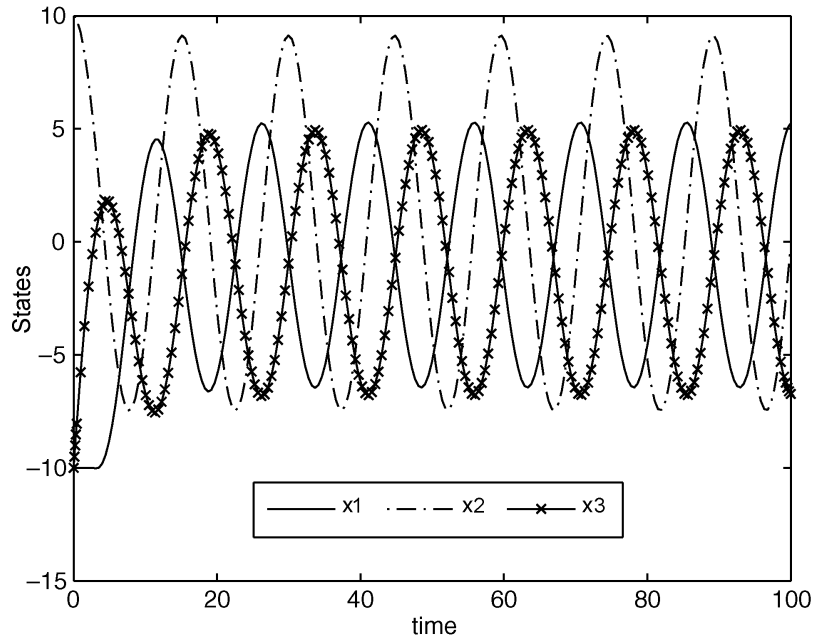

(a)

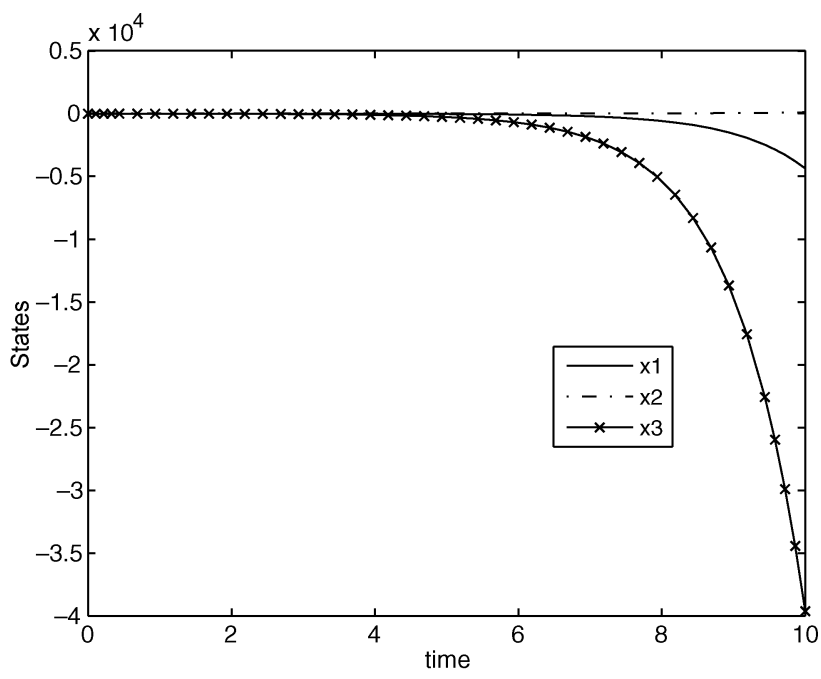

(b)

Fig. 1. Transient behavior of the projection neural network in the counter example. (a) $\lambda=1$. (b) $\lambda=-1$.

Lemma 1: Suppose $f: R^{n} \rightarrow R$ is differentiable and pseudoconvex on $\Omega$. Then $x^{*} \in \Omega \subset R^{n}$ satisfies

$$
\nabla f\left(x^{*}\right)^{T}\left(x-x^{*}\right) \geq 0 \quad \forall x \in \Omega
$$

if and only if $x^{*}$ is a minimum of $f(x)$ in $\Omega$.

Let $P_{\Omega}(\cdot)$ be the projection operator defined by (3). It has the following properties.

Lemma 2 [1, pp. 9-10]: For any $u \in R^{n}$ and any $v \in \Omega \subset$ $R^{n}$

$$
\left(P_{\Omega}(u)-u\right)^{T}\left(v-P_{\Omega}(u)\right) \geq 0 .
$$

For any $u, v \in R^{n}$

$$
\left\|P_{\Omega}(u)-P_{\Omega}(v)\right\| \leq\|u-v\| .
$$

Note that $\operatorname{VI}(F, \Omega)$ does not always have a solution. For existence conditions for the solution of $\operatorname{VI}(F, \Omega)$, one may refer to [1]. Throughout this paper, we assume $\Omega^{*}$ is nonempty and there exists a finite $x^{*} \in \Omega^{*}$.

\section{PSEUdomonotone VARIATIONAL INEQUALITIES AND PSEUDOCONVEX OPTIMIZATION}

\section{A. Existing Stability Results}

Let us first recall some existing results related to our efforts. In [31], Noor considered a more general variational inequality problem, quasi-variational inequality problem, which includes VI in (1) as a special case. When applied to VI, his stability results about the dynamic system (4) read as follows.

- If $F(x)$ is pseudomonotone and Lipschitz continuous, then the dynamic system (4) with $\lambda>0$ is stable in the Lyapunov sense and globally converges to the solution set of (1).

- If $F(x)$ is Lipschitz continuous, the dynamic system (4) with $\lambda<0$ globally and exponentially converges to the unique solution of (1).
The correctness of these results is in question. A counter example is presented as follows. Consider the affine mapping

$$
F(x)=M x+q
$$

where

$$
M=\left(\begin{array}{ccc}
1 & 3 & 1 \\
-3 & 0 & 3 \\
1 & -3 & 1
\end{array}\right) \quad q=\left(\begin{array}{c}
-1 \\
1 \\
4
\end{array}\right)
$$

It is easily verified that $F(x)$ is Lipschitz continuous in $R^{3}$ and $M^{T}+M$ is positive semidefinite in $R^{3}$. Hence, $F(x)$ is monotone and consequently pseudomonotone in $R^{3}$. Let $\Omega=\{x \in$ $\left.R^{3} \mid-10 \leq x_{i} \leq 10, i=1,2,3\right\}$ and use the neural dynamic system (4) to solve this VI. Fig. 1 displays the state trajectory of (4) with the initial point $x_{0}=(-10,10,-10)^{T}$ and $\alpha=0.1$. Clearly, when $\lambda=1$ the trajectories exhibit periodic oscillations and when $\lambda=-1$ the trajectories diverge to infinity. In neither case the system converges to an equilibrium point though all required conditions in [31] are satisfied.

The aforementioned example suggests that the pseudomonotonicity of $F$ alone cannot guarantee the global convergence of the projection neural network (4). It motivates us to strengthen this condition to some extend or add some other conditions to ensure the desired properties. These strengthened conditions or additional conditions may include the strong pseudomonotonicity of $F$, the symmetry of $\nabla F$, the boundedness of $\Omega$ and so on, which are considered in Sections III-B and III-C.

\section{B. Symmetric Jacobian Matrix of $F$}

In this section, we focus on $\operatorname{VI}(F, \Omega)$ with the Jacobian matrix of $F$ being symmetric. The results are then applied to the constrained pseudoconvex optimization problems.

Lemma 3 [25]: Assume that $F(x)$ is locally Lipschitz continuous in $R^{n}$. Then, there exists a unique continuous solution $x(t)$ for (4) with $x\left(t_{0}\right)=x_{0}$. Moreover, when $x_{0} \notin \Omega$, the solution 
$x(t)$ will approach $\Omega$ exponentially; when $x_{0} \in \Omega, x(t) \in \Omega$ for $t \geq t_{0}$.

In what follows, we assume that $F(x)$ is continuously differentiable so that $F(x)$ is locally Lipschitz continuous. The following important result regarding solving VI with the projection neural network is due to Xia et al. [27].

Lemma 4 [27, Th. 1]: If the Jacobian matrix of $F(x)$ is symmetric on the bounded set $\Omega$, then the projection neural network in (4) with the initial point $x_{0} \in \Omega$ is stable in the Lyapunov sense and is globally convergent to the solution set of (1). In particular, if (1) has a unique solution, the projection neural network is globally asymptotically stable.

Theorem 1follows from Lemmas 3 and 4 directly.

Theorem 1: If the Jacobian matrix of $F(x)$ is symmetric on the bounded set $\Omega$, then the projection neural network in (4) with any initial point $x_{0} \in R^{n}$ is stable in the Lyapunov sense and is globally convergent to the solution set of (1). In particular, if (1) has a unique solution, the neural network is globally asymptotically stable.

Theorem 2 shows that the boundedness of $\Omega$ in Theorem 1 can be replaced by the pseudomonotonicity of $F$, and in this case, the neural network can not only converge to the solution set of (1), but also converge to an exact solution.

Theorem 2: If $F(x)$ is pseudomonotone on $\Omega$ and $\nabla F(x)$ is symmetric, then the projection neural network in (4) is stable in the sense of Lyapunov and globally convergent to a solution of (1). In particular, if (1) has a unique solution, the projection neural network is globally asymptotically stable.

Proof: Suppose that $x^{*} \in \Omega^{*}$ is a finite solution of (1). Since by Lemma 3 any trajectory $x(t)$ will exponentially approach $\Omega$ when $x\left(t_{0}\right) \notin \Omega$, and will remain in $\Omega$ forever, it suffices to show the stability of the neural network model with $x\left(t_{0}\right) \in \Omega$. Then, $x(t) \in \Omega$ for $t \geq t_{0}$ and $F\left(x^{*}\right)^{T}\left(x-x^{*}\right) \geq$ $0, \forall t \geq t_{0}$. By the pseudomonotonicity of $F(x)$, we have

$$
F(x)^{T}\left(x-x^{*}\right) \geq 0
$$

Consider the following function on $\Omega$ :

$$
V(x)=\int_{0}^{1}\left(x-x^{*}\right)^{T} F\left(x^{*}+s\left(x-x^{*}\right)\right) d s
$$

where $x^{*} \in \Omega^{*}$. Since $\nabla F(x)$ is symmetric, $\nabla V(x)=F(x)$ [32]. It follows that $V(x)$ is pseudoconvex on $\Omega$ because $F(x)$ is pseudomonotone on $\Omega$ [32]. Moreover, since $x^{*}$ is a solution to $\operatorname{VI}(F, \Omega)$, by Lemma $1, x^{*}$ is a minimum of $V(x)$ in $\Omega$. Then

$$
V(x) \geq V\left(x^{*}\right)=0 \text {. }
$$

Now consider the following Lyapunov function:

$$
V_{1}(x)=\alpha V(x)+\frac{1}{2}\left\|x-x^{*}\right\|^{2} .
$$

Clearly, $V_{1}(x)>0, \forall x \in \Omega$ and $x \neq x^{*}$, and its gradient is given as $\nabla V_{1}(x)=\alpha F(x)+x-x^{*}$. In Lemma 2, let $u=$ $x-\alpha F(x)$ and $v=x^{*}$, then we get

$$
\left(P_{\Omega}(x-\alpha F(x))-x+\alpha F(x)\right)^{T}\left(x^{*}-P_{\Omega}(x-\alpha F(x))\right) \geq 0
$$

which follows:

$$
\begin{aligned}
&\left(P_{\Omega}(x-\alpha F(x))-x\right.+\alpha F(x))^{T} \\
& \cdot\left(P_{\Omega}(x-\alpha F(x))-x+x-x^{*}\right) \leq 0
\end{aligned}
$$

and

$$
\begin{aligned}
& \left(\alpha F(x)+x-x^{*}\right)^{T}\left(P_{\Omega}(x-\alpha F(x))-x\right) \\
& \quad \leq-\left\|P_{\Omega}(x-\alpha F(x))-x\right\|^{2}-\alpha F(x)^{T}\left(x-x^{*}\right) \\
& \quad \leq-\left\|P_{\Omega}(x-\alpha F(x))-x\right\|^{2} .
\end{aligned}
$$

It is to say

$$
\frac{d V_{1}}{d t}=\nabla V_{1}(x)^{T} \frac{d x}{d t} \leq-\lambda\left\|P_{\Omega}(x-\alpha F(x))-x\right\|^{2} \leq 0
$$

and $d V_{1}(x) / d t=0$ if and only if $x \in \Omega^{*}$. Thus, the neural network is stable in the sense of Lyapunov.

Since $V_{1} \geq\left\|x-x^{*}\right\|^{2} / 2$, any level set of $V_{1}$ is bounded. It follows that for any initial point $x\left(t_{0}\right) \in \Omega$, there exists a convergent subsequence $\left\{x\left(t_{k}\right)\right\}$ such that

$$
\lim _{k \rightarrow \infty} x\left(t_{k}\right)=\hat{x}, \quad \text { where } \hat{x} \in \Omega^{*} .
$$

Finally, define a Lyapunov function again

$$
\hat{V}_{1}(x)=\int_{0}^{1}(x-\hat{x})^{T} F(\hat{x}+s(x-\hat{x})) d s+\frac{1}{2}\|x(t)-\hat{x}\|^{2} .
$$

It is easy to see that $\hat{V}(x)$ decreases along the trajectory of (4) and satisfies $\hat{V}(\hat{x})=0$. Therefore, for any $\varepsilon>0$, there exists $q>0$ such that, for all $t \geq t_{q}$

$$
\|x(t)-\hat{x}\|^{2} / 2 \leq \hat{V}(x(t)) \leq \hat{V}\left(x\left(t_{q}\right)\right)<\varepsilon .
$$

Therefore, $\lim _{t \rightarrow \infty} x(t)=\hat{x}$. It follows that the projection neural network in (4) is globally convergent to a solution of (1). In particular, if (1) has a unique solution, the projection neural network is globally asymptotically stable.

Remark 1: It is observed from the proof of Theorem 2 that the convergence rate of the neural network (4) will increase when $\lambda$ increases.

In the special case when $F$ is monotone, we have the following corollary which follows from Theorem 2 directly.

Corollary 1: If $F(x)$ is monotone on $\Omega$ and $\nabla F(x)$ is symmetric, then the projection neural network in (4) is stable in the sense of Lyapunov and globally convergent to the solution set of (1). In particular, if (1) has a unique solution, the projection neural network is globally asymptotically stable.

A similar result to corollary appears in [28, Th. 2.1]. However, corollary only requires the monotonicity of $F$ on $\Omega$, while the counterpart in [28] requires the monotonicity of $F$ in $R^{n}$, which excludes many applications. For example, in the one-dimensional (1-D) case, let $F=x^{2}$ and $\Omega=[1,2]$. Clearly, $F$ 
is monotone on $\Omega$, but not monotone on $R$. One cannot assert the global convergence and asymptotical stability of the neural network (4) at $x^{*}=1$ by the result in [28]; but can do so by Corollary 1.

Theorem 3: Suppose that $F(x)$ is strongly pseudomonotone on $\Omega$ and $\nabla F(x)$ is symmetric. If there exists a constant $\delta>0$ such that

$$
V(x) \leq \delta\left\|x-x^{*}\right\|^{2} \quad \forall x \in \Omega
$$

where $x^{*} \in \Omega^{*}$ and $V(x)$ is defined by (9), then the projection neural network in (4) is globally exponentially convergent to $x^{*}$.

Proof: First, since $F$ is strongly pseudomonotone on $\Omega, \Omega^{*}$ consists of only one solution $x^{*}$. Similar to the argument stated in the beginning of the proof of Theorem 2, it suffices to show the exponential stability of the neural network with $x\left(t_{0}\right) \in \Omega$. Then $x(t) \in \Omega$ for $t \geq t_{0}$ and $F\left(x^{*}\right)^{T}\left(x-x^{*}\right) \geq 0, \forall t \geq t_{0}$. By the strong pseudomonotonicity of $F(x)$, we have

$$
F(x)^{T}\left(x-x^{*}\right) \geq \gamma\left\|x-x^{*}\right\|^{2} .
$$

Consider the Lyapunov function $V_{1}(x)$ in the analysis of Theorem 2. Then

$$
\frac{1}{2}\left\|x-x^{*}\right\|^{2} \leq V_{1}(x) \leq\left(\frac{1}{2}+\alpha \delta\right)\left\|x-x^{*}\right\|^{2} .
$$

In Theorem 2 we have obtained

$$
\begin{aligned}
(\alpha F(x)+ & \left.x-x^{*}\right)^{T}\left(P_{\Omega}(x-\alpha F(x))-x\right) \\
& \leq-\left\|P_{\Omega}(x-\alpha F(x))-x\right\|^{2}-\alpha F(x)^{T}\left(x-x^{*}\right) .
\end{aligned}
$$

It follows:

$\left(\alpha F(x)+x-x^{*}\right)^{T}\left(P_{\Omega}(x-\alpha F(x))-x\right) \leq-\alpha \gamma\left\|x-x^{*}\right\|^{2} \leq 0$.

It is to say

$$
\frac{d V_{1}(x(t))}{d t}=\nabla V_{1}(x)^{T} \frac{d x}{d t} \leq-\alpha \gamma \lambda\left\|x-x^{*}\right\|^{2} \leq-\beta V_{1}(x(t))
$$

where

$$
\beta=\frac{\alpha \gamma \lambda}{(1 / 2)+\alpha \delta}>0
$$

It follows:

$$
V_{1}(x(t)) \leq V_{1}\left(x\left(t_{0}\right)\right) \exp \left(-\beta\left(t-t_{0}\right)\right)
$$

and

$\left\|x(t)-x^{*}\right\| \leq \sqrt{2 V_{1}\left(x\left(t_{0}\right)\right)} \exp \left(-\beta\left(t-t_{0}\right) / 2\right) \quad \forall t \geq t_{0}$. The projection neural network is globally exponentially stable.

Note that for some problems condition (10) in Theorem 3 can be satisfied. For example, let $F(x)=2 x$ and $\Omega=[1,3]$ where $x \in R$. Then $x^{*}=1$ and $V(x)=\alpha\left(x^{2}-1\right)$. Clearly (10) holds.
However, in general, this condition is difficult to be satisfied. For another example, let $F(x)=-2 x$ and $\Omega=[1,3]$. Then $x^{*}=3$ and $V(x)=\alpha\left(9-x^{2}\right)$. There exists no $\delta$ such that (10) holds. The Corollary 2 gives weaker conditions than those in Theorem 3 , and consequently, concludes weaker results.

Corollary 2: Suppose that $F(x)$ is strongly pseudomonotone on $\Omega$ and $\nabla F(x)$ is symmetric. If $\|F(x)\|$ has an upper bound on $\Omega$, then the projection neural network is globally asymptotically stable at the unique solution $x^{*}$ of (1), and its convergence behavior can be described by

$$
\left\|x(t)-x^{*}\right\| \leq \sqrt{\frac{1}{a+b\left(t-t_{0}\right)}} \quad \forall t \geq t_{0}
$$

where $a$ and $b$ are two positive constants.

Proof: As $F(x)$ is strongly pseudomonotone, there is only one solution $x^{*}$ to (1). From Theorem 2 , the neural network is globally asymptotically stable at $x^{*}$. In the proof of Theorem 3 we have obtained

$$
\frac{d V_{1}(x(t))}{d t} \leq-\alpha \gamma \lambda\left\|x-x^{*}\right\|^{2} \leq 0 \quad \forall t \geq t_{0}
$$

which implies

$$
\left\{x(t) \mid t \geq t_{0}\right\} \subset S=\left\{x \in \Omega \mid V_{1}(x(t)) \leq V_{1}\left(x\left(t_{0}\right)\right)\right\} .
$$

Since $V_{1}(x) \geq(1 / 2)\left\|x-x^{*}\right\|^{2}$, set $S$ is bounded. Note that $S$ may not be convex as $V_{1}(x)$ may not be pseudoconvex. Since $x^{*} \in S$, there exists a constant $L_{1}>0$ such that $\left\|x-x^{*}\right\| \leq L_{1}$ for $t \geq t_{0}$. Moreover, since $V(x)$ defined by (9) is continuously differentiable, there exists $\xi$ between $x$ and $x^{*}$ such that

$$
V(x)=V\left(x^{*}\right)+\left(x-x^{*}\right)^{T} F(\xi)=\left(x-x^{*}\right)^{T} F(\xi) .
$$

As $\Omega$ is convex, we have $\xi \in \Omega$ and $\|F(\xi)\| \leq L_{2}$ by assumption, where $L_{2}>0$ is a constant. It follows $V(x) \leq L_{2}\left\|x-x^{*}\right\|$ and

$$
V_{1}(x) \leq\left(L_{1} / 2+\alpha L_{2}\right)\left\|x-x^{*}\right\|
$$

Hence

$$
d V_{1}(x(t)) / d t \leq-\alpha \gamma \lambda\left\|x-x^{*}\right\|^{2} \leq-\beta V_{1}(x(t))^{2}
$$

where

$$
\beta=\frac{\alpha \gamma \lambda}{\left(L_{1} / 2+\alpha L_{2}\right)^{2}}>0 .
$$

It follows:

$$
V_{1}(x(t)) \leq \frac{1}{1 / V_{1}\left(x\left(t_{0}\right)\right)+\beta\left(t-t_{0}\right)} \quad \forall t \geq t_{0}
$$

which yields (11), where $a=1 /\left(2 V_{1}\left(x\left(t_{0}\right)\right)\right)$ and $b=\beta / 2$.

It is noticed that in proving Theorems 1,2 , and 3 and Corollary 2 , the symmetry of $\nabla F$ plays a critical role. This fact motivates us to solve a class of constrained optimization problems considering that the Hessian matrix of any objective function 
is always symmetric if it is continuous. Consider the following optimization problem:

$$
\min f(x) \text { subject to } x \in \Omega \text {. }
$$

If $f(x)$ is pseudoconvex and twice continuously differentiable, from Lemma 1, (12) is equivalent to VI in (1) with $F=\nabla f$. Then the following projection neural network can be used to solve (12):

$$
\frac{d x}{d t}=\lambda\left\{-x+P_{\Omega}(x-\alpha \nabla f(x))\right\} .
$$

The Corollary 3 follows from Theorems 2 and 3 and Corollary 2.

Corollary 3: Assume that $f(x)$ is pseudoconvex and twice continuously differentiable on the closed convex set $\Omega$. Then the projection neural network in (13) is stable in the sense of Lyapunov and globally convergent to a solution of (12). Moreover, the following hold.

- If $\nabla f$ is strongly pseudomonotone on $\Omega$ and there exists $\delta>0$ such that $f(x) \leq \delta\left\|x-x^{*}\right\|^{2}$, where $x^{*}$ is the unique solution of (12), then the neural network in (13) is globally exponentially stable.

- If $\nabla f$ is strongly pseudomonotone on $\Omega$ and $\|\nabla f(x)\|$ has an upper bound on $\Omega$, then the projection neural network in (13) is globally asymptotically stable at the unique solution of (12), while the convergence rate is upper bounded by (11).

Remark 2: One of the important classes of pseudoconvex optimization problems are the quadratic fractional problems in the following form:

$$
\begin{aligned}
& \min f(x)=\frac{x^{T} Q x+a^{T} x+a_{0} x}{b^{T} x+b_{0}} \\
& \text { subject to } x \in R^{n} \in X=\left\{x \mid b^{T} x+b_{0}>0\right\}
\end{aligned}
$$

where $Q$ is an $n \times n$ symmetric matrix, $a, b \in R^{n}$, and $a_{0}$, $b_{0} \in R$. It is well known (e.g., [35]) that $f$ is pseudoconvex on $X$ when $Q$ is positive-semidefinite. Conditions for $f$ being pseudoconvex on $X$ when $Q$ is not positive-semidefinite are discussed in [36]. Specially, when $b=0$, problem (14) is reduced to the classic quadratic programming problem, and when $Q=0$ it is reduced to the so called linear fractional problem, which is, of course, pseudoconvex on $X$ [37].

\section{Asymmetric Jacobian Matrix of F}

When the Jacobian matrix of $F(x)$ is asymmetric, it is in general difficult to formulate a function (e.g., $V(x)$ in the proof of Theorem 2) such that its gradient is $F$. Moreover, when $F$ is assumed to be only pseudomonotone but not monotone, many nice properties of its Jacobian matrix such as the positive semidefiniteness and positive definiteness are lost. These are major difficulties in proving the stabilities of the projection neural network. Nevertheless, under some stronger conditions, we find that the neural network in (4) possesses globally exponential convergence property, which is stated in Theorem 4.

Theorem 4: Assume that $F(x)$ is Lipschitz continuous on $\Omega$ with constant $L$ and strongly pseudomonotone on $\Omega$ with constant $\gamma>0$. If $\gamma>2 L$, then the projection neural network in (4) is globally exponentially stable.

Proof: Since $F(x)$ is strongly pseudomonotone, there is only one solution $x^{*}$ to $\operatorname{VI}(F, \Omega)$. According to Lemma 3, it suffices to show the exponential stability of the neural network with $x\left(t_{0}\right) \in \Omega$. Let $x=P_{\Omega}(x-\alpha F(x))$ and $x^{*}=x^{*}$ in (1), we have

$$
F\left(x^{*}\right)^{T}\left(P_{\Omega}(x-\alpha F(x))-x^{*}\right) \geq 0 .
$$

The strong pseudomonotonicity of $F(x)$ implies

$$
\begin{aligned}
F\left(P_{\Omega}(x-\alpha F(x))\right)^{T} & \left(P_{\Omega}(x-\alpha F(x))-x^{*}\right) \\
& \geq \gamma\left\|P_{\Omega}(x-\alpha F(x))-x^{*}\right\|^{2} .
\end{aligned}
$$

By letting $u=x-\alpha F(x)$ and $v=x^{*}$ in (8), we get

$$
\left(P_{\Omega}(x-\alpha F(x))-x+\alpha F(x)\right)^{T}\left(x^{*}-P_{\Omega}(x-\alpha F(x))\right) \geq 0 .
$$

Multiplying $\alpha$ to both sides of (15) and adding the resultant inequality to (16) gives

$$
\begin{aligned}
& {\left[P_{\Omega}(x-\alpha F(x))-x+\alpha F(x)-\alpha F\left(P_{\Omega}(x-\alpha F(x))\right)\right]^{T}} \\
& \cdot\left[x^{*}-P_{\Omega}(x-\alpha F(x))\right] \geq \alpha \gamma\left\|P_{\Omega}(x-\alpha F(x))-x^{*}\right\|^{2}
\end{aligned}
$$

which follows:

$$
\begin{aligned}
& {\left[x^{*}-x+\alpha F(x)-\alpha F\left(x^{*}\right)\right]^{T}\left[x^{*}-P_{\Omega}(x-\alpha F(x))\right]} \\
& \quad-\left\|P_{\Omega}(x-\alpha F(x))-x^{*}\right\|^{2} \\
& \quad+\alpha\left[F\left(x^{*}\right)-F\left(P_{\Omega}(x-\alpha F(x))\right)\right]^{T} \\
& \quad \cdot\left[x^{*}-P_{\Omega}(x-\alpha F(x))\right] \geq \alpha \gamma\left\|P_{\Omega}(x-\alpha F(x))-x^{*}\right\|^{2}
\end{aligned}
$$

and

$$
\begin{aligned}
(1+ & \alpha \gamma)\left\|P_{\Omega}(x-\alpha F(x))-x^{*}\right\|^{2} \\
\leq & {\left[x^{*}-x+\alpha F(x)-\alpha F\left(x^{*}\right)\right]^{T}\left[x^{*}-P_{\Omega}(x-\alpha F(x))\right] } \\
& +\alpha\left[F\left(x^{*}\right)-F\left(P_{\Omega}(x-\alpha F(x))\right)\right]^{T} \\
& \times\left[x^{*}-P_{\Omega}(x-\alpha F(x))\right] \\
\leq & {\left[x^{*}-x+\alpha F(x)-\alpha F\left(x^{*}\right)\right]^{T}\left[x^{*}-P_{\Omega}(x-\alpha F(x))\right] } \\
& +\alpha L\left\|P_{\Omega}(x-\alpha F(x))-x^{*}\right\|^{2} .
\end{aligned}
$$

By noting that $(1+\alpha(\gamma-L))>0$, we have

$$
\begin{aligned}
& \left\|P_{\Omega}(x-\alpha F(x))-x^{*}\right\| \\
& \quad \leq \frac{1}{1+\alpha(\gamma-L)}\left\|x^{*}-x+\alpha F(x)-\alpha F\left(x^{*}\right)\right\| \\
& \quad \leq \frac{1+\alpha L}{1+\alpha(\gamma-L)}\left\|x^{*}-x\right\| .
\end{aligned}
$$

Consider the function

$$
V=\frac{1}{2}\left\|x(t)-x^{*}\right\|^{2} \quad \forall x(t) \in \Omega .
$$


Then

$$
\begin{aligned}
\frac{d V}{d t} & =\left(x-x^{*}\right)^{T} \frac{d x}{d t} \\
& =\lambda\left\{\left(x-x^{*}\right)^{T}\left(P_{\Omega}(x-\alpha F(x))-x\right)\right\} \\
& =\lambda\left\{\left(x-x^{*}\right)^{T}\left(P_{\Omega}(x-\alpha F(x))-x^{*}\right)-\left\|x-x^{*}\right\|^{2}\right\} \\
& \leq \lambda\left\{\left\|x-x^{*}\right\|\left\|P_{\Omega}(x-\alpha F(x))-x^{*}\right\|-\left\|x-x^{*}\right\|^{2}\right\} \\
& \leq \frac{\lambda(1+\alpha L)}{1+\alpha(\gamma-L)}\left\|x-x^{*}\right\|^{2}-\lambda\left\|x-x^{*}\right\|^{2} \\
& =-\beta\left\|x-x^{*}\right\|^{2}
\end{aligned}
$$

where

$$
\beta=\frac{\lambda \alpha(\gamma-2 L)}{1+\alpha(\gamma-L)}>0
$$

Therefore

$$
\left\|x(t)-x^{*}\right\| \leq\left\|x\left(t_{0}\right)-x^{*}\right\| e^{-\beta\left(t-t_{0}\right)} \quad \forall t \geq t_{0} .
$$

The projection neural network in (4) is globally exponentially stable.

\section{COMPONENTWISE PSEUdOMONOTONE VARIATIONAL INEQUALITIES}

Definition 4: A function $F: R^{n} \rightarrow R^{n}$ is said to be componentwise pseudomonotone (CPM) on $\Omega$ if, for every pair of points $x, y \in \Omega$ and all $i=1, \ldots, n$

$$
F_{i}(x)\left(y_{i}-x_{i}\right) \geq 0 \Longrightarrow F_{i}(y)\left(y_{i}-x_{i}\right) \geq 0 .
$$

$F$ is said to be strictly CPM on $\Omega$ if, for every pair of points $x, y \in \Omega$ and all $i=1, \ldots, n$

$$
F_{i}(x)\left(y_{i}-x_{i}\right) \geq 0 \Longrightarrow F_{i}(y)\left(y_{i}-x_{i}\right)>0 \quad \forall x_{i} \neq y_{i}
$$

and strongly CPM on $\Omega$ if there exists a constant $\gamma>0$ such that for every pair of points $x, y \in \Omega$ and all $i=1, \ldots, n$

$$
F_{i}(x)\left(y_{i}-x_{i}\right) \geq 0 \Longrightarrow F_{i}(y)\left(y_{i}-x_{i}\right) \geq \gamma\left(x_{i}-y_{i}\right)^{2} \text {. }
$$

The above CPM definitions are easily seen as listed in an order from weak to strong. Similarly, we can define the componentwise monotonicity (or CM), strictly CM and strongly CM of the mapping $F$, which again are listed in an order from weak to strong. Moreover, CPM is a generalization of CM. Specifically, CM implies CPM, strict CM implies strict CPM and strong CM implies strong CPM; but not vice versa.

Remark 3: In general, the pseudomonotonicity does not imply the CPM. For example, the mapping $F(x)=$ $\left(-\left(x_{2}-2\right) / x_{1}^{2}, 1 / x_{1}\right)^{T}$ is known to be pseudomonotone on $\Omega=\left\{x \in R^{2} \mid 1 \leq x_{i} \leq 3, i=1,2\right\}$ since $F$ is the gradient of the function $f(x)=\left(x_{2}-2\right) / x_{1}$, which is pseudoconvex on $\Omega$ according to Remark 2. Take $x=(2,3)^{T}$ and $y=(1,1)^{T}$. Then $F_{1}(x)\left(y_{1}-x_{1}\right)=(1 / 4)>0$, but $F_{1}(y)\left(y_{1}-x_{1}\right)=-1<0$. Therefore, $F$ is not CPM on $\Omega$. The CPM does not imply the pseudomonotonicity either. Consider the mapping $F(x)=\left(-x_{1},-2 x_{2}\right)^{T}$. It is CPM on $\Omega=\left\{x \in R^{2} \mid 1 \leq x_{i} \leq 3, i=1,2\right\}$, but not pseudomonotone on $\Omega$ by simple reasoning.

It will be shown that under different CPM conditions, the projection neural network in (4) also possesses desired global convergence to the solution set of $\operatorname{VI}(F, \Omega)$ and global asymptotic stability or global exponential stability. Without loss of generality, we hereafter assume $\lambda=1$ in (4) for convenience. It is readily found that all the convergence results presented hereafter hold when $\lambda \neq 1$ but $\lambda>0$. Two Lemmas should be introduced first.

Lemma 5: If $x^{*} \in \Omega$ is a solution of $\operatorname{VI}(F, \Omega)$, where $\Omega$ is a box set, then $F_{i}\left(x^{*}\right)\left(x_{i}-x_{i}^{*}\right) \geq 0$ for all $i \in I=\{1, \ldots, n\}$ and all $x \in \Omega$.

Proof: Otherwise, without loss of generality, suppose there exists one point $\hat{x} \in \Omega$ such that $F_{i}\left(x^{*}\right)\left(\hat{x}_{i}-x_{i}^{*}\right)<0, \forall i \in$ $I_{0} \subset I$, and $F_{i}\left(x^{*}\right)\left(\hat{x}_{i}-x_{i}^{*}\right) \geq 0, \forall i \in I_{1}=I \backslash I_{0}$, where $I_{0} \neq \emptyset$. Consider a vector $\tilde{x}$ with its components chosen as $\tilde{x}_{i}=\hat{x}_{i}, \forall i \in I_{0}$ and $\tilde{x}_{i}=x_{i}^{*}, \forall i \in I_{1}$. Because $\Omega$ is a box-type set and both $\hat{x}$ and $x^{*}$ are in $\Omega, \tilde{x}$ must be in $\Omega$. Then

$$
\begin{aligned}
F\left(x^{*}\right)^{T}\left(\tilde{x}-x^{*}\right)= & \sum_{i \in I_{0}} F_{i}\left(x^{*}\right)\left(\tilde{x}_{i}-x_{i}^{*}\right) \\
& +\sum_{i \in I_{1}} F_{i}\left(x^{*}\right)\left(\tilde{x}_{i}-x_{i}^{*}\right) \\
= & \sum_{i \in I_{0}} F_{i}\left(x^{*}\right)\left(\hat{x}_{i}-x_{i}^{*}\right) \\
& +\sum_{i \in I_{1}} F_{i}\left(x^{*}\right)\left(x_{i}^{*}-x_{i}^{*}\right) \\
= & \sum_{i \in I_{0}} F_{i}\left(x^{*}\right)\left(\hat{x}_{i}-x_{i}^{*}\right)<0
\end{aligned}
$$

which contradicts the fact that $x^{*}$ is a solution of $\operatorname{VI}(F, \Omega)$.

Lemma 6: If $F(x)$ is strictly CPM on a box set $\Omega$, then $\mathrm{VI}(F, \Omega)$ has at most one solution.

Proof: Suppose $\operatorname{VI}(F, \Omega)$ has two distinct solution $x^{*}$ and $y^{*}$ in $\Omega$. By Lemma $5, \forall x \in \Omega$ we have

$$
\begin{aligned}
F\left(x^{*}\right)^{T}\left(x-x^{*}\right) \geq 0 & \Rightarrow F_{i}\left(x^{*}\right)\left(x_{i}-x_{i}^{*}\right) \geq 0 & & \forall i=1, \ldots, n \\
& \Rightarrow F_{i}(x)\left(x_{i}-x_{i}^{*}\right)>0 & & \forall i=1, \ldots, n \\
& \Rightarrow F(x)^{T}\left(x-x^{*}\right)>0 . & &
\end{aligned}
$$

Substituting $x=y^{*}$ into aforementioned resultant inequality yields

$$
F\left(y^{*}\right)^{T}\left(x^{*}-y^{*}\right)<0
$$

which contradicts to the fact that $y^{*}$ is also a solution of VI.

Lemma 7: Assume $x(t) \in \Omega, \forall t \in\left[t_{0}, \infty\right)$, where $\Omega$ is a box set. Then there exists a continuous function $\mu_{i}: R^{n} \rightarrow R$ for all $i=1, \ldots, n$ and $t \geq t_{0}$ such that

1) the following system is equivalent to the neural network in (4):

$$
\frac{d x_{i}}{d t}=-\mu_{i}(x) F_{i}(x), \quad i=1, \ldots, n ;
$$

2) $\mu_{i}(x) \geq 0$;

3) if $\mu_{i}(x)=0$, then $x_{i}=x_{i}^{*}$, where $x^{*} \in \Omega^{*}$.

Proof: Write (4) in the scalar form

$$
\frac{d x_{i}}{d t}=P_{\Omega_{i}}\left(x_{i}-\alpha F_{i}(x)\right)-x_{i}, \quad i=1, \ldots, n
$$

where $\Omega_{i}=\left[l_{i}, u_{i}\right]$. 
Case 1) If $x_{i}(t)-\alpha F_{i}(x(t)) \in\left[l_{i}, u_{i}\right], \forall t \in T_{1}$, where $T_{1} \subset$ $\left[t_{0}, \infty\right)$ denotes any time period, (19) simplifies to

$$
\frac{d x_{i}}{d t}=-\alpha F_{i}(x)
$$

From the above system, it is seen that the $i$ th component of the equilibrium point $x^{*}$ of system (20) is attained within time period $T_{1}$ if and only if $F_{i}\left(x^{*}\right)=0$.

Case 2) If $x_{i}(t)-\alpha F_{i}(x(t))>u_{i}, \forall t \in T_{2}$, where $T_{2} \subset$ $\left[t_{0}, \infty\right)$ denotes any time period, (19) becomes

$$
\frac{d x_{i}}{d t}=u_{i}-x_{i} \geq 0
$$

since $x_{i}(t) \in \Omega_{i}$. Considering that $x_{i}(t)-$ $\alpha F_{i}(x(t))>u_{i}$, we have $F_{i}(x(t))<0$. Then there exists a continuous function $\beta: R^{n} \rightarrow R$ such that $\beta(x) \geq 0$ and the following system is equivalent to (21) for $t \in T_{2}$ :

$$
\frac{d x_{i}}{d t}=-\beta(x) F_{i}(x) \geq 0 .
$$

Clearly, whenever $\beta(x)=0$, then $d x_{i} / d t=0$, and the $i$ th component of the equilibrium point $x^{*}$ is attained. Moreover, $x_{i}^{*}=u_{i}$ from (21). Conversely, if the $i$ th component of the equilibrium point $x^{*}$ of system (22) is attained within time period $T_{2}$, we must have $\beta(x)=0$ since $F_{i}(x(t))<0, \forall t \in T_{2}$.

Case 3) If $x_{i}(t)-\alpha F_{i}(x(t))<l_{i}, \forall t \in T_{3}$, where $T_{3} \subset$ $\left[t_{0}, \infty\right)$ denotes any time period, (19) becomes

$$
\frac{d x_{i}}{d t}=l_{i}-x_{i} \leq 0
$$

since $x_{i}(t) \in \Omega_{i}$. Considering that $x_{i}(t)-$ $\alpha F_{i}(x(t))<l_{i}$, we have $F_{i}(x(t))>0$. Then there exists a continuous function $\gamma: R^{n} \rightarrow R$ such that $\gamma(x) \geq 0$ and the following system is equivalent to (23) for $t \in T_{3}$ :

$$
\frac{d x_{i}}{d t}=-\gamma(x) F_{i}(x) \leq 0
$$

Clearly, whenever $\gamma(x)=0$, then $d x_{i} / d t=0$, and the $i$ th component of the equilibrium point $x^{*}$ is attained. Moreover, $x_{i}^{*}=l_{i}$ from (23). Conversely, if the $i$ th component of the equilibrium point $x^{*}$ of system (24) is attained within time period $T_{3}$, we must have $\gamma(x)=0$ since $F_{i}(x(t))>0, \forall t \in T_{3}$.

In summary, (19) can be equivalently put in the form of (18) with

$$
\mu_{i}(x)= \begin{cases}\alpha>0, & \forall t \in T_{1} \\ \beta(x) \geq 0, & \forall t \in T_{2} \\ \gamma(x) \geq 0, & \forall t \in T_{3}\end{cases}
$$

Moreover, $\mu_{i}(x)=0$ implies that $x_{i}^{*}$ is attained. Now consider at some instant $\hat{t}, x_{i}(\hat{t})-\alpha F_{i}(x(\hat{t}))=u_{i}$, then $\hat{t}$ could only be the intersection of $T_{1}$ and $T_{2}$. It follows from (20)-(22) that

$\frac{d x_{i}(\hat{t})}{d t}=-\alpha F_{i}(x(\hat{t}))=u_{i}-x_{i}(\hat{t})=-\beta(x(\hat{t})) F_{i}(x(\hat{t}))$.

Thus $\alpha=\beta(x(\hat{t}))$. Similarly, we can see that if at some instant $\bar{t}, x_{i}(\bar{t})-\alpha F_{i}(x(\bar{t}))=l_{i}$, then $\bar{t}$ could only be the intersection of $T_{1}$ and $T_{3}$ and $\alpha=\gamma(x(\bar{t}))$. Therefore, the aforementioned function $\mu_{i}(x)$ is continuous in $x$.

Theorem 5: If $F(x)$ is CPM on $\Omega$, where $\Omega$ is a box set, then the projection neural network in (4) is stable in the Lyapunov sense and is globally convergent to a solution of (1). In particular, it is globally asymptotically stable if (1) has a unique solution.

Proof: By Lemma 3, without loss of generality we assume $x\left(t_{0}\right) \in \Omega$ and thus $x(t) \in \Omega$ for $t \geq t_{0}$. Suppose $x^{*}$ is a finite solution of (1), by Lemma 5 and the CPM of $F(x)$ we have

$$
\begin{aligned}
F\left(x^{*}\right)^{T}\left(x-x^{*}\right) \geq 0 & \Rightarrow F_{i}\left(x^{*}\right)\left(x_{i}-x_{i}^{*}\right) \geq 0 \\
& \Rightarrow F_{i}(x)\left(x_{i}-x_{i}^{*}\right) \geq 0
\end{aligned}
$$

for all $i=1, \ldots, n$ and $x \in \Omega$. Now consider the following Lyapunov function:

$$
V(x)=\frac{1}{2}\left\|x(t)-x^{*}\right\|^{2}, \quad t \geq t_{0} .
$$

By Lemma 7

$$
\frac{d V}{d t}=\left(x-x^{*}\right)^{T} \frac{d x}{d t}=-\sum_{i=1}^{n} \mu_{i}(x)\left(x_{i}-x_{i}^{*}\right) F_{i}(x) \leq 0
$$

and, hence

$$
x(t) \in \Omega_{0}=\left\{x \in \Omega \mid V(x(t)) \leq V\left(x\left(t_{0}\right)\right)\right\} .
$$

Clearly, $\Omega_{0}$ is bounded. It follows from the LaSalle invariant set theorem that the trajectory $x(t)$ will converge to the largest invariant subset of the following set:

$$
S=\left\{x(t) \in \Omega_{0} \mid d V / d t=0\right\} .
$$

On one hand, if $d x / d t=0$, from (18), $d V / d t=0$. On the other hand, if $d V / d t=0$, then either $\mu_{i}(x)=0$ or $\left(x_{i}-x_{i}^{*}\right) F_{i}(x)=$ 0 for all $i=1, \ldots, n$, since both of them are nonnegative for all $i=1, \ldots, n$. Clearly, $\left(x_{i}-x_{i}^{*}\right) F_{i}(x)=0$ means either $x_{i}=x_{i}^{*}$ or $F_{i}(x)=0$, which further implies $d x_{i} / d t=0$. According to Lemma 7, $\mu_{i}(x)=0$ also implies $d x_{i} / d t=0$. Therefore, $d V / d t=0 \Leftrightarrow d x / d t=0$.

According to the Lyapunov theorem, there exists a convergent subsequence $\left\{x\left(t_{k}\right)\right\}$ such that

$$
\lim _{k \rightarrow \infty} x\left(t_{k}\right)=\hat{x} \quad \text { where } \quad \hat{x} \in \Omega^{*} .
$$


Finally, define a Lyapunov function again

$$
\hat{V}(x)=\frac{1}{2}\|x(t)-\hat{x}\|^{2}, \quad x(t) \in \Omega
$$

It is easy to see that $\hat{V}(x)$ decreases along the trajectory of (4) and satisfies $\hat{V}(\hat{x})=0$. Therefore, for any $\varepsilon>0$, there exists $q>0$ such that, for all $t \geq t_{q}$

$$
\|x(t)-\hat{x}\|^{2} / 2=\hat{V}(x(t)) \leq \hat{V}\left(x\left(t_{q}\right)\right)<\varepsilon
$$

and, thus, $\lim _{t \rightarrow \infty} x(t)=\hat{x}$. Hence, the neural network (4) is globally convergent to a solution of (1). In particular, if (1) has a unique solution, the projection neural network is globally asymptotically stable.

Theorem 6: If $F(x)$ is strongly CPM on $\Omega$, where $\Omega$ is a box set, then the neural network in (4) is globally exponentially stable.

Proof: Since $F(x)$ is strongly CPM, according to Lemma 6, (1) has a unique solution $x^{*}$. By Lemma 3, it suffices to show the exponential stability of the neural network model with $x\left(t_{0}\right) \in \Omega$. By Lemma 5 and the strong CPM of $F(x)$ we have

$$
\begin{aligned}
F\left(x^{*}\right)\left(x-x^{*}\right) \geq 0 & \Rightarrow F_{i}\left(x^{*}\right)\left(x_{i}-x_{i}^{*}\right) \geq 0 \\
& \Rightarrow F_{i}(x)\left(x_{i}-x_{i}^{*}\right) \geq \gamma\left(x_{i}-x_{i}^{*}\right)^{2}
\end{aligned}
$$

for all $i=1, \ldots, n$ and $x \in \Omega$. Consider the function $V$ defined in the proof of Theorem 5 , and

$$
V_{0}=\sum_{i \in I_{0}(t)}\left(x_{i}-x_{i}^{*}\right)^{2} / 2 \quad V_{1}=\sum_{i \in I_{1}(t)}\left(x_{i}-x_{i}^{*}\right)^{2} / 2
$$

where $I_{0}(t)=\left\{i \in I \mid \mu_{i}(x(t))=0\right\}, I_{1}(t)=I \backslash I_{0}(t)$ and $I=\{1, \ldots, n\}$. Then $V=V_{0}+V_{1}$. Clearly, whenever $x \notin \Omega^{*}$, $I_{1} \neq \emptyset$. According to Lemma 7 , we have

$$
\frac{d V_{0}}{d t}=\sum_{i \in I_{0}(t)}\left(x_{i}-x_{i}^{*}\right) \frac{d x_{i}}{d t}=-\sum_{i \in I_{0}(t)} \mu_{i}(x)\left(x_{i}-x_{i}^{*}\right) F_{i}(x)
$$

and

$$
\begin{aligned}
\frac{d V_{1}}{d t} & =\sum_{i \in I_{1}(t)}\left(x_{i}-x_{i}^{*}\right) \frac{d x_{i}}{d t}=-\sum_{i \in I_{1}(t)} \mu_{i}(x)\left(x_{i}-x_{i}^{*}\right) F_{i}(x) \\
& \leq-\sum_{i \in I_{1}(t)} \mu_{i}(x) \gamma\left(x_{i}-x_{i}^{*}\right)^{2} \leq-\sum_{i \in I_{1}(t)} \varsigma(t)\left(x_{i}-x_{i}^{*}\right)^{2} \\
& =-2 \varsigma(t) V_{1}
\end{aligned}
$$

where $\varsigma(t)=\min _{i \in I_{1}(t)}\left\{\mu_{i}(x) \gamma\right\}>0$, as $\gamma>0$ and $\mu_{i}(x)>0$ for $i \in I_{1}(t)$. Note for $i \in I_{0}(t), \mu_{i}(x)=0$ implies $x_{i}=x_{i}^{*}$, hence both $V_{0}$ and $d V_{0} / d t$ are equal to zero at time $t$. Then

$$
\frac{d V}{d t}=\frac{d V_{1}}{d t} \leq-2 \varsigma(t) V_{1}=-2 \varsigma(t) V
$$

It follows:

$$
\left\|x(t)-x^{*}\right\| \leq\left\|x\left(t_{0}\right)-x^{*}\right\| e^{-\varsigma(t)\left(t-t_{0}\right)} \quad \forall t \geq t_{0} .
$$

The projection neural network in (4) is globally exponentially stable at $x^{*}$.

Remark 4: Since VI contains many problems as special cases, such as the fixed-point problem and the linear and nonlinear complementarity problems, besides the constrained optimization problems, these problems are also solvable by using the neural network in (4) under the conditions discussed in Sections III and IV. Take the nonlinear complementarity problem (NCP) for example. Find a vector $x \in R^{n}$ such that

$$
x \geq 0 \quad F(x) \geq 0 \quad x^{T} F(x)=0
$$

where $F: R^{n} \rightarrow R^{n}$ is a differentiable vector-valued mapping. It is well known (e.g., see [2] and [4]) that $\operatorname{VI}(F, \Omega)$ with $\Omega$ taking the nonnegative orthant of $R^{n}$ (denoted by $R_{+}^{n}$ ) is equivalent to the NCP defined in (25). Thus, the projection neural network (4) can be applied to solve the NCP, and the corresponding dynamic system becomes

$$
\frac{d x}{d t}=\lambda\{-x+g(x-\alpha F(x))\}
$$

where the activation function $g(x)=\left[g_{1}\left(x_{1}\right), \ldots, g_{n}\left(x_{n}\right)\right]^{T}$ and $g_{i}\left(x_{i}\right)=\max \left\{x_{i}, 0\right\}$. Most of the conditions regarding the stability of the neural network (4) discussed heretofore in the paper apply to the projection neural network (26), and will not be repeated here.

\section{NUMERICAL EXAMPLES}

In order to demonstrate the effectiveness and performance of the projection neural network in solving pseudomonotone variational inequalities and pseudoconvex optimization problems, we give several illustrative examples in this section.

Example 1: Consider a VI (1) with

$$
F(x)=\left(\begin{array}{l}
x_{1}+x_{2}+x_{3}+x_{4}-4 x_{2} x_{3} x_{4} \\
x_{1}+x_{2}+x_{3}+x_{4}-4 x_{1} x_{3} x_{4} \\
x_{1}+x_{2}+x_{3}+x_{4}-4 x_{1} x_{2} x_{4} \\
x_{1}+x_{2}+x_{3}+x_{4}-4 x_{1} x_{2} x_{3}
\end{array}\right)
$$

and $\Omega=\left\{x \in R^{4} \mid 1 \leq x_{i} \leq 5, i=1, \ldots, 4\right\}$. The problem has only one solution $x^{*}=(5,5,5,5)^{T}$. Obviously $\nabla F(x)$ is symmetric. It is easy to see that $F(x)$ is not monotone on $\Omega$; however, it is not easy to verify that it is pseudomonotone on $\Omega$. Actually, in general, it is a very difficult task to check the pseudomonotonicity of a mapping in practice. We here employ the Monte Carlo approach according to the definition of pseudomonotonicity: Generate a large number of pairs of points $x$ and $y$ uniformly in $\Omega$ satisfying $F(x)^{T}(y-x) \geq 0$ and then check if $F(y)^{T}(y-x) \geq 0$. For this problem, one million pairs of such points have been tested and all satisfy (6). Thus, it is reasonable to assert that $F(x)$ is pseudomonotone on $\Omega$. 


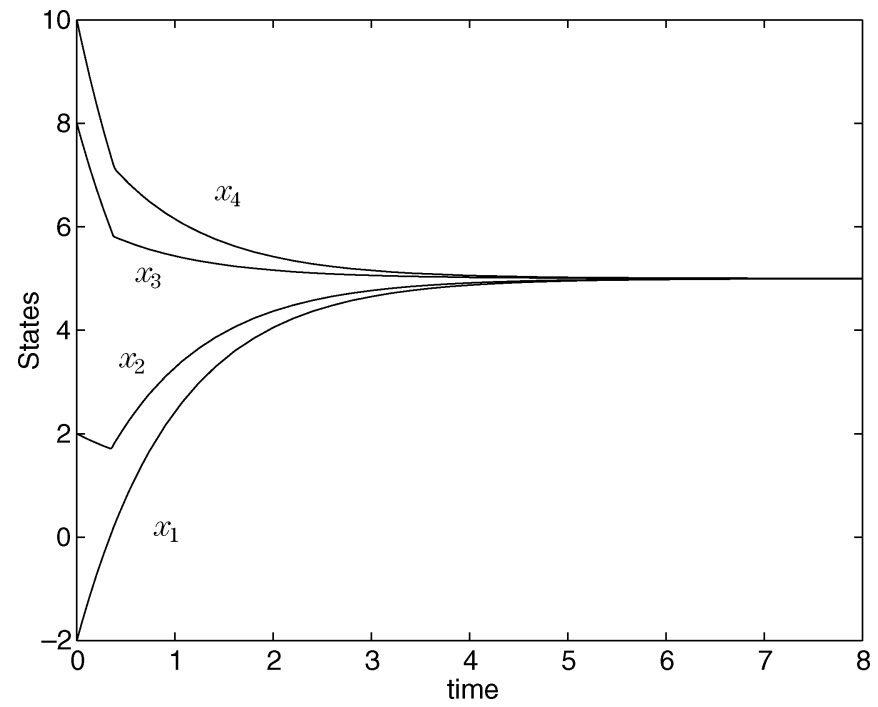

Fig. 2. Transient behavior of the neural network in Example 1.

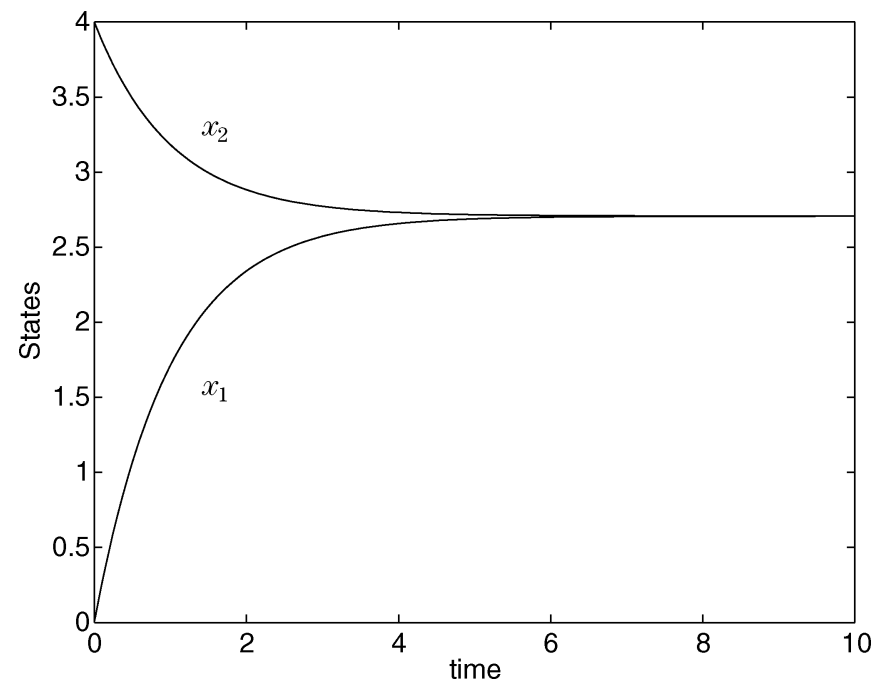

Fig. 3. Transient behavior of the neural network with initial point $x_{0}=(0,4)^{T}$ in Example 2 .

Thus, the conditions in both Theorems 1 and 2 are satisfied. We then use the projection neural network in (4) to solve this problem. All simulation results show that the projection neural network is globally asymptotically stable at $x^{*}$. For instance, when $\lambda=\alpha=1$, Fig. 2 shows that the trajectory of (4) with the initial point $x_{0}=(-2,2,8,10)^{T}$ converges to $x^{*}$.

Example 2: Consider a two-dimensional (2-D) VI with a spherical constraint. Let

$$
F(x)=\left(\begin{array}{c}
0.5 x_{1} x_{2}-2 x_{2}-10^{7} \\
-4 x_{1}+0.1 x_{2}^{2}-10^{7}
\end{array}\right)
$$

and $\Omega=\left\{x \in R^{2} \mid\left(x_{1}-2\right)^{2}+\left(x_{2}-2\right)^{2} \leq 1\right\}$. It is easy to see that $\nabla F(x)$ is asymmetric and $F(x)$ is not monotone on $\Omega$. By conducting a similar testing procedure to that descried

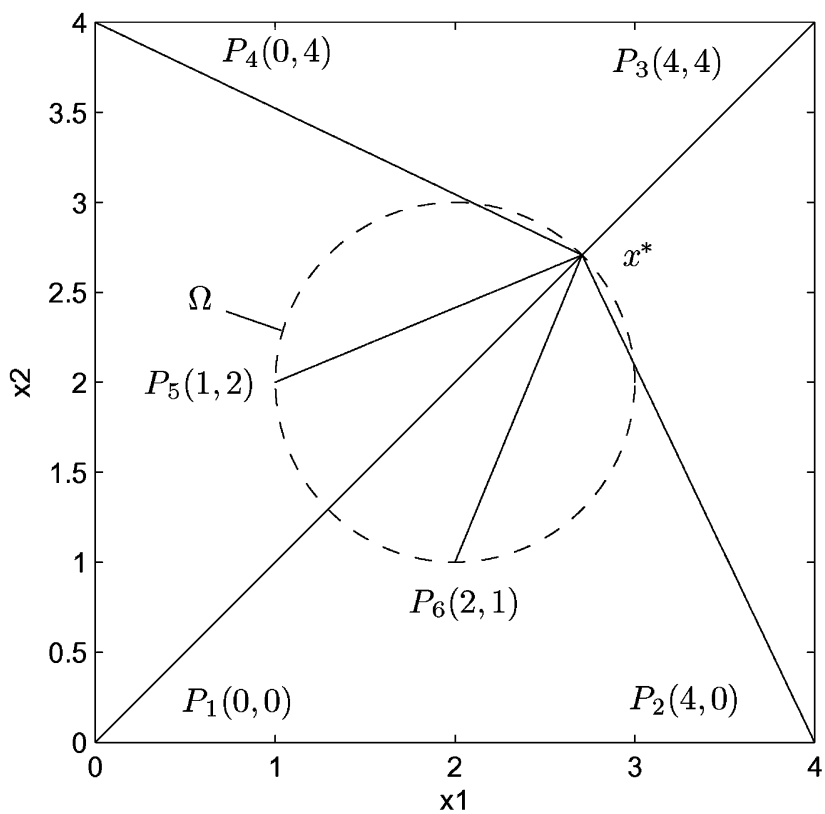

Fig. 4. Transient behavior of the neural network with six initial points in Example 2.

in Example 1, we are confident that $F(x)$ is strongly pseudomonotone on $\Omega$ with constant $\gamma=11$, and Lipschitz continuous on $\Omega$ with constant $L=5$. We then use the projection neural network in (4) to solve the aforementioned problem. According to Theorem 4, the neural network should be globally exponentially stable. All simulations confirm this point and the neural network always globally converges to the unique solution $x^{*}=(2.707,2.707)^{T}$. For instance, when $\lambda=\alpha=1$, Fig. 3 displays the trajectories of (4) with the initial point $x_{0}=$ $(0,4)^{T}$. Fig. 4 displays the trajectories of (4) with six different initial points $P_{1}(0,0), P_{2}(4,0), P_{3}(4,4), P_{4}(0,4), P_{5}(1,2)$, and $P_{6}(2,1)$, among which the last two points are located in $\Omega$ and the others are not. It can be seen that all trajectories converge to $x^{*}$ exponentially.

Example 3 is about the componentwise pseudomonotone VI.

Example 3: Let us consider the $\mathrm{VI}(F, \Omega)$ with

$$
F(x)=\left(\begin{array}{c}
-2 x_{1}^{3}-e^{x_{1}+x_{2}+x_{3}-x_{4}-x_{5}} \\
-5 x_{2}^{3}-e^{x_{1}+x_{2}+x_{3}-x_{4}-x_{5}} \\
-x_{3}^{3}-e^{x_{1}+x_{2}+x_{3}-x_{4}-x_{5}} \\
-x_{4}^{3}-5 x_{1} x_{2} x_{3} x_{4} x_{5} \\
-x_{5}^{3}-2 x_{1} x_{2} x_{3} x_{4} x_{5}
\end{array}\right)
$$

and $\Omega=\left\{x \in R^{5} \mid 1 \leq x_{i} \leq 5, i=1, \ldots, 5\right\}$. It is seen that $F$ is not pseudomonotone (one may take $x=(1.8,1.7,3.0,3.7,3.0)^{T}$ and $y=(2.4,4.6,4.9,1.2,4.3)^{T}$ to verify this point). However, $F$ is strictly CPM. Therefore, Theorem 5 applies here. We use the projection neural network (4) to solve this problem. All simulations show that the neural network is globally asymptotically stable at the unique optimum $x^{*}=(5,5,5,5,5)^{T}$. For instance, when $\lambda=\alpha=1$, Fig. 5 shows the transient behavior of the neural network with the initial point $x_{0}=(15,10,0,-5,-10)^{T}$. 


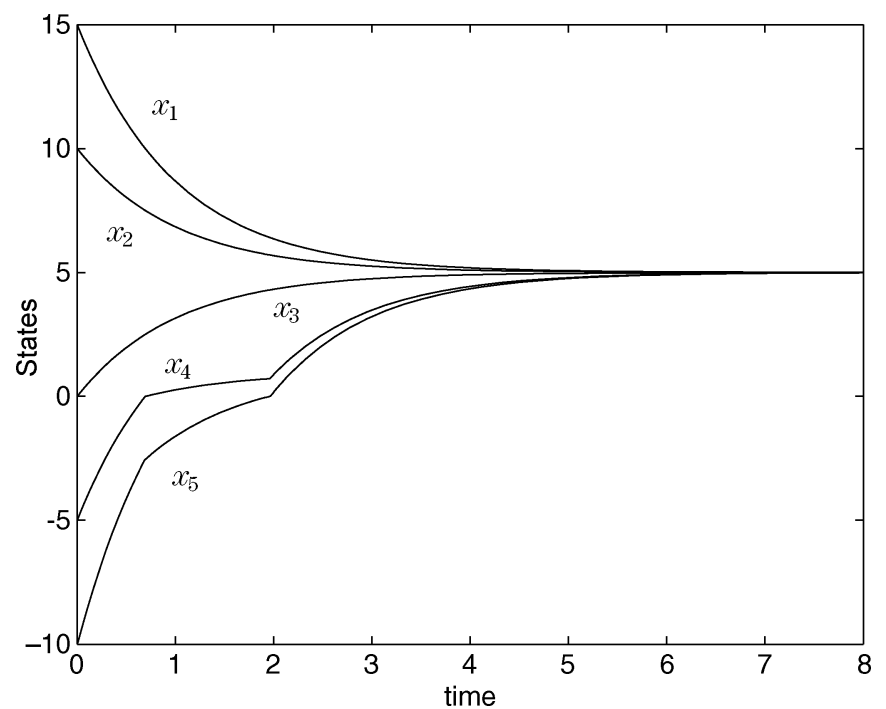

Fig. 5. Transient behavior of the neural network in Example 3.

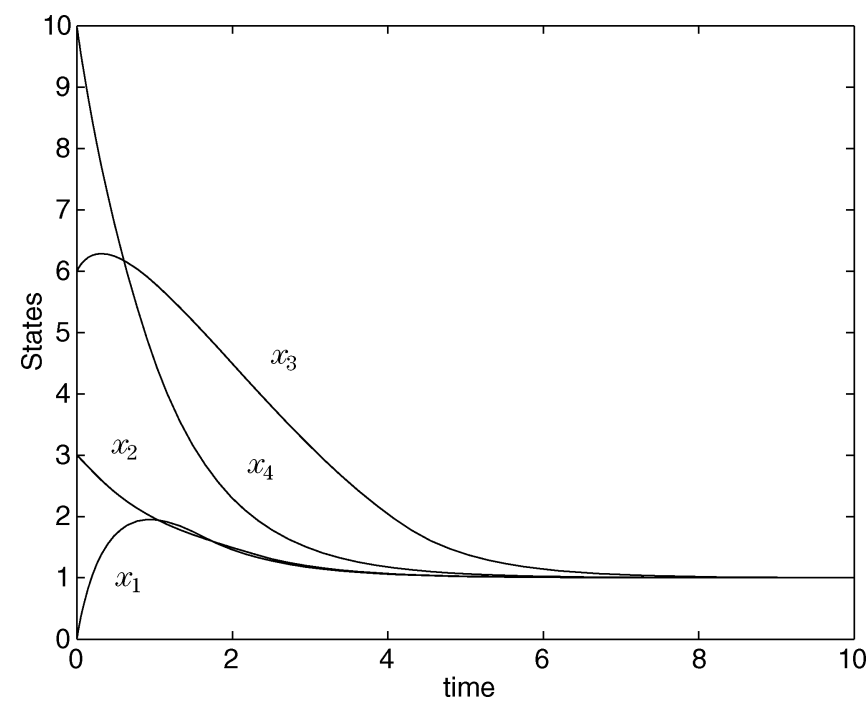

Fig. 6. Transient behavior of the neural network in Example 4.

Example 4: We now use the projection neural network to solve a pseudoconvex optimization problem. Consider the quadratic fractional programming problem in (14) with

$$
\begin{aligned}
Q & =\left(\begin{array}{cccc}
5 & -1 & 2 & 0 \\
-1 & 5 & -1 & 3 \\
2 & -1 & 3 & 0 \\
0 & 3 & 0 & 5
\end{array}\right) \quad a=\left(\begin{array}{c}
1 \\
-2 \\
-2 \\
1
\end{array}\right) \quad b=\left(\begin{array}{l}
2 \\
1 \\
1 \\
0
\end{array}\right) \\
a_{0} & =-2 \quad b_{0}=4 .
\end{aligned}
$$

It is easily verified that $Q$ is symmetric and positive-definite in $R^{4}$, and consequently $f$ is pseudoconvex on $X=\{x \in$ $\left.R^{4} \mid b^{T} x+b_{0}>0\right\}$. We minimize $f$ over $\Omega=\left\{x \in R^{4} \mid 1 \leq\right.$ $\left.x_{i} \leq 10, i=1, \ldots, 4\right\} \subset X$ by using the neural network in
(4) with $F(x)=\nabla f(x)$ which can be written in the following explicit form:

$$
\nabla f(x)=\frac{\left(b^{T} x+b_{0}\right)(2 Q x+a)-b\left(x^{T} Q x+a^{T} x+a_{0}\right)}{\left(b^{T} x+b_{0}\right)^{2}} .
$$

This problem has a unique solution $x^{*}=(1,1,1,1)^{T}$ in $\Omega$. All simulations show the projection neural network in (4) is globally asymptotically stable at $x^{*}$. For instance, Fig. 6 shows that the trajectory of the neural network with $\lambda=\alpha=1$ and the initial point $x_{0}=(0,3,6,10)^{T}$ converges to $x^{*}$.

A counter example in Section IV shows that the pseudomonotonicity of a mapping solely does not ensure the global convergence of the projection neural network in (4) to the VI (1); the next example, however, shows that the neural network may have global convergence when the mapping is neither pseudomonotone nor componentwise pseudomonotone.

Example 5: Let us consider the NCP defined by (25) with

$$
F(x)=\left(\begin{array}{c}
3 x_{1}^{2}+2 x_{1} x_{2}+2 x_{2}^{2}+x_{3}+3 x_{4}-6 \\
2 x_{1}^{2}+x_{1}+x_{2}^{2}+10 x_{3}+2 x_{4}-2 \\
3 x_{1}^{2}+x_{1} x_{2}+2 x_{2}^{2}+2 x_{3}+9 x_{4}-9 \\
x_{1}^{2}+3 x_{2}^{2}+2 x_{3}+3 x_{4}-3
\end{array}\right)
$$

which has two solutions $x^{*}=(1,0,3,0)^{T}$ and $\hat{x}^{*}=$ $(\sqrt{6} / 2,0,0,(1 / 2))^{T}$. This problem first appeared in [38] and was tested by other researchers [12], [39]. Obviously, $F$ is not monotone in $R_{+}^{4}$ and the Jacobian matrix of $F$ is asymmetric. Furthermore, it is easily verified numerically that $F(x)$ is neither pseudomonotone nor componentwise pseudomonotone in $R_{+}^{4}$, though it is often used to test numerical algorithms that are designed for solving the pseudomonotone VI (see [12] for example). We use the projection neural network in (26) to solve this problem. All simulation results show that the neural network is globally convergent to one of the optima $x^{*}$. For instance, Fig. 7(a) and (b) displays the trajectories of (4) with two initial points $x_{0}^{\prime}=(10,5,10,-10)^{T}$ and $x_{0}^{\prime \prime}=(2,-2,-1,5)^{T}$, respectively. They both converge to $x^{*}$ eventually. Notice that with $x_{0}^{\prime \prime}$ the neural network first converges to the other optimum $\hat{x}^{*}$ and then converges to $x^{*}$ [see Fig. 7(b)]. That is to say, $\hat{x}^{*}$ is not a stable equilibrium point of the neural network (26).

\section{CONCluding REMARKS}

In this paper, we extended the scope of an existing projection neural network, which was originally proposed for solving monotone variational inequalities, to pseudomonotone variational inequalities and pseudoconvex optimization problems. In the case of symmetric Jacobian matrix of the nonlinear mapping, we proved that the projection neural network is globally convergent, globally asymptotically stable, and global exponentially stable, respectively, under pseudomononicity and other conditions. In the case of asymmetric Jacobian matrix of the mapping, we proved that the projection neural network is globally exponentially stable under strong pseudomonotonicity and other conditions. Furthermore, by defining several new pseudomonotonicities (i.e., various componentwise pseudomonotonicties), we proved the global convergence and 


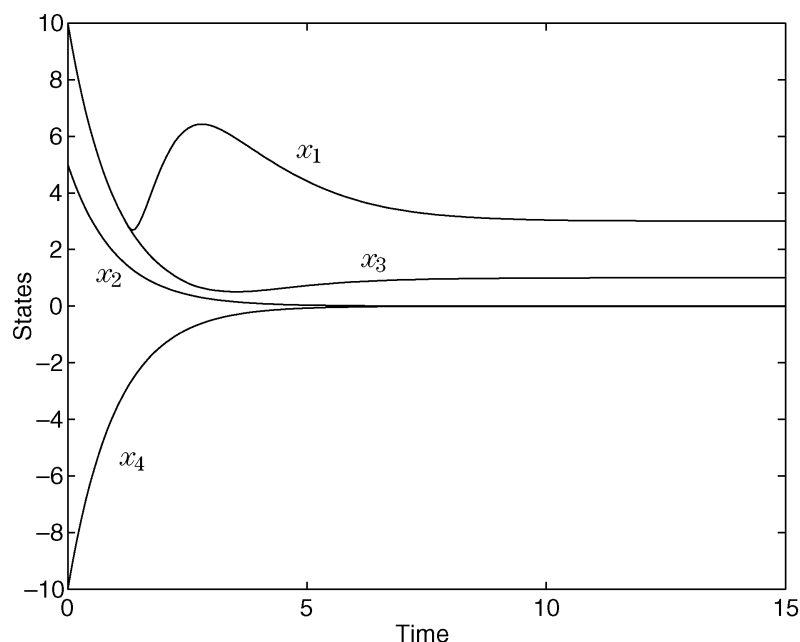

(a)

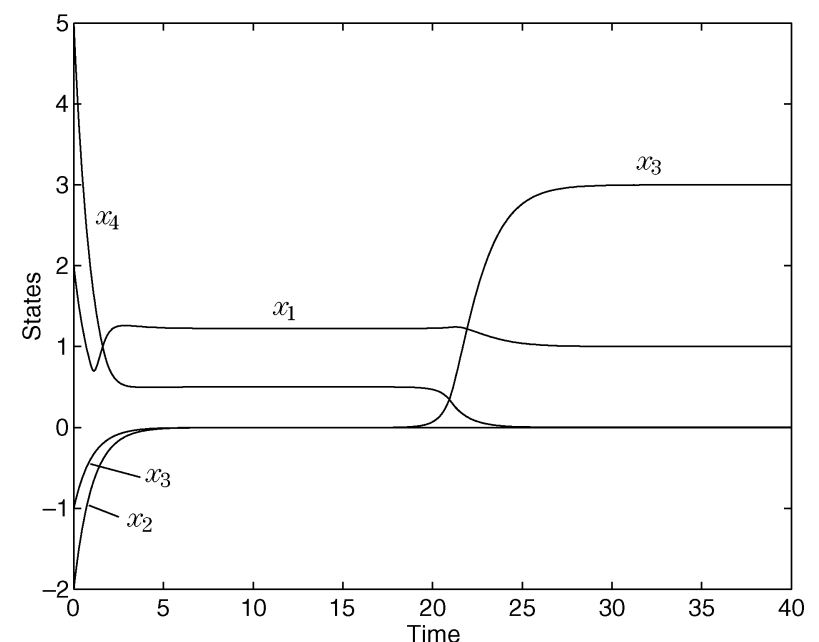

(b)

Fig. 7. Transient behavior of the neural network with two initial points in Example 5. (a) $x_{0}^{\prime}=(10,5,10,-10)^{T}$. (b) $x_{0}^{\prime \prime}=(2,-2,-1,5)^{T}$.

global exponential stability of the projection neural network under these conditions. Simulation studies demonstrated the effectiveness of the projection neural network and thus validated the theoretical results. Moreover, the last illustrative example suggests that the potential of the projection neural network has been far less than fully explored, which calls for further investigations in improving the stability conditions.

\section{REFERENCES}

[1] D. Kinderlehrer and G. Stampcchia, An introduction to variational inequalities and their applications. New York: Academic, 1980.

[2] R. W. Cottle, F. Giannessi, and J. L. Lions, Variational Inequalities and Complementarity Problems: Theorey and Applications. New York: Wiley, 1980.

[3] L. Vandenberghe, B. L. De Moor, and J. Vandewalle, "The generalized linear complementarity problem applied to the complete analysis of resistive piecewise-linear circuits," IEEE Trans. Circuits Syst., vol. 36, no. 11, pp. 1382-1391, Nov. 1989.

[4] P. T. Harker and J. S. Pang, "Finite-dimensional variational inequality and nonlinear complementarity problems: a survey of theory, algorithms and applications," Math. Program., vol. 48, pp. 161-220, 1990.

[5] T. L. Friesz, D. H. Bernstein, N. J. Mehta, R. L. Tobin, and S. Ganjlizadeh, "Day-to-day dynamic network disequilibria and idealized traveler information systems," Oper. Res., vol. 42, no. 6, pp. 1120-1136, 1994.

[6] M. C. Ferris and J. S. Pang, "Engineering and economic applications of complementarity problems," Soc. Ind. Appl. Math. (SIAM) Rev., vol. 39, pp. 669-713, 1997.

[7] M. Fukushima, "Equivalent differentiable optimization problems and descent methods for asymmetric variational inequality problems," Math. Program., vol. 53, pp. 99-110, 1992.

[8] T. Larsson and M. Patriksson, "A class of gap functions for variational inequalities," Math. Program., vol. 64, pp. 53-79, 1994.

[9] M. V. Solodov and P. Tseng, "Modified projection-type methods for momotone variational inequalities," Soc. Ind. Appl. Math. (SIAM) J. Control Optim., vol. 2, pp. 1814-1830, 1996.

[10] B. S. He, Z. H. Yang, and X. M. Yuan, "An approximate proximalextragradient type method for monotone variational inequalities," $J$. Math. Anal. Appl., vol. 300, pp. 362-374, 2004.

[11] N. E. Farouq, "Pseudomonotone variational inequalities: convergence of proximal methods," J. Optim. Theory Appl., vol. 109, no. 2, pp. 311-326, May 2001.

[12] Y. J. Wang, N. H. Xiu, and C. Y. Wang, "Unified framework of extragradient-type methods for pseudomonotone variational inequalities," $J$. Optim. Theory Appl., vol. 111, no. 3, pp. 641-656, Dec. 2001.

[13] M. A. Noor, "Modified projection method for pseudomonotone variational inequalities," Appl. Math. Lett., vol. 15, pp. 315-320, 2002.
[14] T. Yoshikawa, Foundations of Robotics: Analysis and Control. Cambridge, MA: MIT Press, 1990.

[15] B. Kosko, Neural Networks for Signal Processing. Englewood Cliffs, NJ: Prentice-Hall, 1992.

[16] J. J. Hopfield and D. W. Tank, "'Neural' computation of Decisions in optimization problems," Biol. Cybern., vol. 52, pp. 141-152, 1985.

[17] D. W. Tank and J. J. Hopfield, "Simple neural optimization networks: an A/D converter, signal decision circuit, and a linear programming circuit," IEEE Trans. Circuits Syst., vol. CS-33, no. 5, pp. 533-541, May 1986.

[18] M. P. Kennedy and L. O. Chua, "Neural networks for nonlinear programming," IEEE Trans. Circuits Syst., vol. 35, no. 5, pp. 554-562, May 1988.

[19] A. Rodríguez-Vázquez, R. Domínguez-Castro, A. Rueda, J. L. Huertas, and E. Sánchez-Sinencio, "Nonlinear switched-capacitor neural networks' for optimization problems," IEEE Trans. Circuits Syst., vol. 37, no. 3, pp. 384-397, Mar. 1990.

[20] J. Wang, "A deterministic annealing neural network for convex programming," Neural Netw., vol. 7, no. 4, pp. 629-641, 1994.

[21] Y. Xia and J. Wang, "A recurrent neural network for nonlinear convex optimization subject to nonlinear inequality constraints," IEEE Trans. Circuits Syst. I, Reg. Papers, vol. 51, no. 7, pp. 1385-1394, Jul. 2004.

[22] Y. Xia, G. Feng, and J. Wang, "A recurrent neural network with exponential convergence for solving convex quadratic program and linear piecewise equations," Neural Netw., vol. 17, no. 7, pp. 1003-1015, 2004.

[23] Y. Xia and J. Wang, "Recurrent neural networks for solving nonlinear convex programs with linear constraints," IEEE Trans. Neural Netw., vol. 16, no. 2, pp. 379-386, Mar. 2005.

[24] _ _ "A recurrent neural network for solving linear projection equations," Neural Netw., vol. 13, no. 3, pp. 337-350, 2000.

[25] — "On the stability of globally projected dynamical systems," $J$. Optim. Theory Appl., vol. 106, no. 1, pp. 129-150, 2000.

[26] X. Liang and J. Si, "Global exponential stability of neural networks with globally Lipschitz continuous activations and its application to linear variational inequality problem," IEEE Trans. Neural Netw., vol. 12, no. 2, pp. 349-359, Mar. 2001.

[27] Y. Xia, H. Leung, and J. Wang, "A projection neural network and its application to constrained optimization problems," IEEE Trans. Circuits Syst. I, Reg. Papers, vol. 49, no. 4, pp. 447-458, Apr. 2002.

[28] Y.Xia, "Further results on global convergence and stability of globally projected dynamical systems," J. Optim. Theory Appl., vol. 122, no. 3, pp. 627-649, 2004.

[29] M. A. Noor, "A Wiener-Hopf dynamical system for variational inequalities," New Zealand J. Math., vol. 31, pp. 173-182, 2002.

[30] X. Hu and J. Wang, "Global stability of a recurrent neural network for solving pseudomonotone variational inequalities," in Proc. IEEE Int. Symp. Circuits Syst., Island of Kos, Greece, May 21-24, 2006, pp. $755-758$.

[31] M. A. Noor, "Implicit dynamical systems and quasi variational inequalities," Appl. Math. Comput., vol. 134, pp. 69-81, 2003. 
[32] S. Karamardian and S. Schaible, "Seven kinds of monotone maps," $J$. Optim. Theory Appl., vol. 66, no. 1, pp. 37-46, 1990.

[33] N. Hadjisavvas and S. Schaible, "On strong pseudomonotonicity and (semi)strict quasimonotonicity," J. Optim. Theory Appl., vol. 79, no. 1, pp. 139-155, 1993.

[34] R. K. Miller and A. N. Michel, Ordinary Differential Equations. New York: Academic, 1982.

[35] M. Avriel, W. E. Diewert, S. Schaible, and I. Zang, Generalized concavity, Mathematical Concepts and Methods in Science and Engineering. New York: Plenum, 1988, vol. 36.

[36] A. Cambini, J. P. Crouzeix, and L. Martein, "On the pseudoconvexity of a quadratic fractional function," Optimization, vol. 51, no. 4, pp. 677-687, 2002.

[37] M. S. Bazaraa, D. S. Hanifand, and C. M. Shetty, Nonlinear Programming: Theory and Algorithms, 2nd ed. New York: Wiley, 1993.

[38] M. Kojima and S. shindo, "Extensions of Newton and quasi-Newton methods to systems of $P C^{1}$ equations," J. Oper. Res. Soc. Japan, vol. 29, pp. 352-374, 1986.

[39] J. S. Pang and A. Gabriel, "NE/SQP: a robust algorithm for the nonlinear complementarity problem," Math. Program., vol. 60, pp. 295-337, 1993.

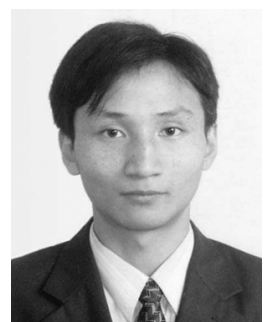

Xiaolin Hu (S'01) received the B.E. and M.E. degrees in automotive engineering from Wuhan University of Technology, Wuhan, China, in 2001 and 2004, respectively. Currently, he is working towards the Ph.D. degree in the Department of Automation and Computer-Aided Engineering, the Chinese University of Hong Kong, Hong Kong, China.

His current research interests include neural networks and their applications in intelligent control.

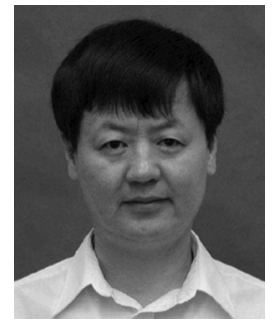

Jun Wang (S'89-M'90-SM'93) received the B.S. degree in electrical engineering and the M.S. degree in systems engineering from Dalian University of Technology, Dalian, Liaoning, China, and the Ph.D. degree in systems engineering from Case Western Reserve University, Cleveland, OH, in 1982, 1985, 1991, respectively.

He is a Professor at the Department of Automation and Computer-Aided Engineering, Chinese University of Hong Kong, Hong Kong, China. He was an Associate Professor at the University of North Dakota, Grand Forks, until 1995. His current research interests include neural networks and their engineering applications.

Dr. Wang is an Associate Editor of the IEEE TRANSACTIONS ON NeURAL NeTworks and IEEE TRANSACTIONS ON SySTEMS, MAN, AND Cybernetics-PART B: Cybernetics. He is a past President of the Asia Pacific Neural Network Assembly. 